INPLASY

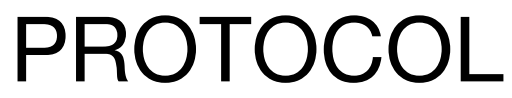

To cite: Zhang et al.

Magnesium valproate adjuvant therapy on patients with dementia: A protocol for systematic review and metaanalysis. Inplasy protocol

2021110038. doi:

10.37766/inplasy2021.11.0038

Received: 12 November 2021

Published: 12 November 2021

Corresponding author:

Hongbin Sun

shb001369x@163.com

Author Affiliation:

Sichuan academy of medical sciences and Sichuan

provincial people's hospital.

Support: Administration of Traditional Chinese Medicine of Sichuan Province, China (grant no.2016C001).

Review Stage at time of this submission: Formal screening of search results against eligibility criteria.

Conflicts of interest:

None declared.

\section{Magnesium valproate adjuvant therapy on patients with dementia: A protocol for systematic review and meta-analysis}

Zhang, CQ1; Zheng, KX²; Sun, LQ33 Sun, HB4.

Review question / Objective: To evaluate the efficacy of magnesium valproate(VPM) in the adjuvant treatment of patients with dementia(PwD).

Participant or population: Adults with dementia (as diagnosed by a clinician, or using any recognized diagnostic criteria) will be included.

Information sources: MEDLINE via PubMed, Cochrane Library, EBSCO, Embase, China National Knowledge(CNKI) and Wan fang databases.

INPLASY registration number: This protocol was registered with the International Platform of Registered Systematic Review and Meta-Analysis Protocols (INPLASY) on 12 November 2021 and was last updated on 17 November 2021 (registration number INPLASY2021110038).

\section{INTRODUCTION}

Review question / Objective: To evaluate the efficacy of magnesium valproate(VPM) in the adjuvant treatment of patients with dementia(PwD).
Condition being studied: According to US population estimate of people with clinical $A D$ and mild cognitive impairment( $\mathrm{MCl})$, an estimated 6.2 million Americans aged 65 and older are living with AD today. By 2060, number could grow to 13.8 million. 
Dementia affects individuals, their families, and the social economy, its costs estimated at about US\$1 trillion annually. However, there is no pharmacological treatment currently for dementia that can delay or stop the damage and destruction of neurons, which is the reason of Alzheimer's symptoms and make the disease fatal. According to the in vitro and in vivo studies, valproic acid may have neuroprotective effects on PwD, through a variety of potential mechanisms including actions on gamma-aminobutyric acid (GABA) and N-methyl-D-aspartate (NMDA) receptors, prevention of betaamyloid aggregation, decreased beta amyloid and neurotic plaque production, and induction of neurogenesis to ameliorate the symptoms of dementia.On the other hand, magnesium for its ability to affect vascular function in addition to neuronal function. Thus, based on these theories, VPM may be affecting cognitive function in multiple distinct ways.Although most of the experiments showed negative results of Valproic acid for dementia patients, most of these tests used sodium valproate as a single therapy to compare with the placebo group. In China, many controlled studies using magnesium valproate as adjuvant therapy showed positive effects with dementia patients. Hence, current research has found contradictory results on the treatment of magnesium valproate. It requires further investigation and standardized ways to evaluate the effects of magnesium valproate on cognitive function in dementia patients. Currently, there is lack of metaanalysis focusing on cognitive improvement and disease-modifying about VPM-assisted therapy in the current peerreviewed literature. Thus, we aimed to likely analyze the efficacy and safety of VPM adjuvant therapy of PwD based on RCTs.

\section{METHODS}

Search strategy: Online databases which including the MEDLINE via PubMed, Cochrane Library databases, and EBSCO, Embase were comprehensive searched by 2 searchers. We don't have restrictions on language, but the search object was restricted to human. To obtain the search results, the search strategy was conducted by using medical subject headings (Mesh) and term words, such as "Valproate Magnesium"[Mesh], "Valproic acid magnesium," "Magnesium dipropylacetate," "Dementia" [Mesh], "dement*," "Alzheimer*," "Huntington*," and so on(see Supplementary MaterialsAdditional File for full electronic search). Additionally, we carefully screened all references relevant to the included studies to avoid inappropriate omissions.

Participant or population: Adults with dementia (as diagnosed by a clinician, or using any recognized diagnostic criteria) will be included.

Intervention: Magnesium valproate as an adjuvant therapy was the main intervention.

Comparator: Anti-dementia medicines mono therapy.

Study designs to be included: Randomized controlled trials (RCTs) will be included.

Eligibility criteria: VPM as an adjunctive therapy provided to the monotherapy group and the study provided detailed and clear outcome of interest.

Information sources: MEDLINE via PubMed, Cochrane Library, EBSCO, Embase, China National Knowledge(CNKI) and Wan fang databases.

Main outcome(s): Efficacy in cognitive effects (including mean change in MMSE and MOCA score).

Additional outcome(s): Efficacy in psychiatric effects.

Data management: Two authors will independently extract data. Any disagreement will be resolved by discussion until consensus is reached or by consulting a third author. The following data will be extracted: author's name with publication date), sample sizes, mean age, 
Gender, details of participants, diagnostic criteria, treatment and control intervention, duration time, main outcome measures and adverse events (AEs).

Quality assessment / Risk of bias analysis: The guidelines for assessing risk of bias provided in the Cochrane handbooks were used to assess the quality of the included studies, and the domains assessed included random sequence generation, allocation concealment, blinding of the participants and personnel, blinding of the assessors, incomplete outcome data, and selective outcome reporting.

Strategy of data synthesis: Stata $\mathbf{1 6 . 0}$ software will performed for our statistical analyses. Standardized mean difference (SMD) with the $95 \% \mathrm{Cl}$ as an effect size was measured for continuous data. As far as dichotomous data, the risk ratios (RRs) OR odds ratios (ORs) with $95 \% \mathrm{Cl}$ was calculated. Cochran's $Q$ statistic and 12 metric statistics were used to assess the level of heterogeneity. For 12 , if $12>50 \%$, the level of heterogeneity was considered unacceptable, and the data were analyzed with a random-effect model. A fixed-effect model was applied when $12<50 \%$.

Subgroup analysis: We plan to conduct subgroup analyses about duration time of the included trials to evaluate the influences of the following factors on primary outcome.

Sensitivity analysis: If necessary ,a leaveone-out sensitivity analysis will be performed to evaluate the main trials demonstrating a substantial impact on the inter-study heterogeneity. If there is no qualitative change in the combined effect, the results are stable.

Country(ies) involved: China.

Keywords: magnesium valproate, dementia, Meta-Analysis.

Contributions of each author:

Author 1 - Chen qi Zhang.

Author 2 - Kexin Zheng.

Author 3 - Lingqi Sun.

Author 4 - Hongbin Sun. 